\title{
Misiones económicas en Colombia y su incidencia en la educación técnica industrial (1930-1960) ${ }^{*}$
}

DOI: https://doi.org/10.18046/recs.i34.4193

\author{
Economic Missions in Colombia and its Impact on Industrial \\ Technical Education, 1930-1960
}

\author{
Álvaro Acevedo-Tarazona ${ }^{* *}$ \\ Universidad Industrial de Santander (Bucaramanga, Colombia)
}

Dayana Lucía Lizcano-Herrera ${ }^{* * *}$

Universidad Santo Tomás Seccional Bucaramanga (Bucaramanga, Colombia)

\footnotetext{
* Artículo derivado del proyecto de investigación "Estado de excepción, conspiración y represión en Bucaramanga" financiado por la Vicerrectoría de Investigación y Extensión de la Universidad Industrial de Santander (código 2400), y desarrollado entre el 28 de agosto de 2018 y el 19 de julio de 2020. Artículo de investigación recibido el 27.07.2020 y aprobado el 12.01.2021.

${ }^{* *}$ Historiador, Magíster en Historia, Especialista en Filosofía, Doctor en Historia y Posdoctorado en Ciencias de la Educación. Profesor titular de la Universidad Industrial de Santander (Colombia). Director del grupo de investigación Políticas, Sociabilidades y Representaciones Histórico-Educativas. Correo electrónico: tarazona20@gmail.com ORCID: https://orcid.org/oooo-0oo2-3563-9213

*** Historiadora, Magíster en Historia y candidata a Doctora en Historia. Docente de la Universidad Santo Tomás Seccional Bucaramanga (Colombia). Miembro del grupo de investigación Políticas, Sociabilidades y Representaciones Histórico-Educativas. Correo electrónico: mhuisdaliz@hotmail.com ORCID: https://orcid.org/oooo-ooo3-1651-1887
} 


\section{Cómo citar/How to cite}

Acevedo-Tarazona, Álvaro; Lizcano-Herrera, Dayana Lucía (2021). Misiones económicas en Colombia y su incidencia en la educación técnica industrial (1930-1960). Revista CS, 34, 241-264. https://doi.org/10.18046/recs.i34.4193 


\section{Resumen}

Este artículo establece la contribución de algunas misiones económicas en la educación colombiana entre 1930 y 1960, las cuales siguieron las directrices impartidas por las misiones educativas que, de manera simultánea, arribaron al país. Las fuentes de este artículo son los informes oficiales presentados por las misiones y la normatividad existente apoyada con historiografía, lo cual permite determinar aspectos políticos y económicos de Colombia en diferentes momentos. Las misiones Kemmerer (1923, 1930), Currie (1949-1950) y Lebret (1954-1956) diagnosticaron el acontecer económico y social del país, y determinaron las condiciones para emprender procesos de crecimiento a partir del desarrollo de la educación técnica industrial en sus diferentes niveles, para así cumplir con los requerimientos de políticas externas que promovieron el desarrollo de América Latina, lo cual impulsó la formación técnica industrial y la consolidación del sistema educativo en general.

\section{PALABRAS CLAVE:}

desarrollo industrial, enseñanza técnica, relaciones económicas internacionales

This article presents the contribution of some economic missions to the Colombian education between 1930 and 1960, which followed the guidelines given by the educational missions that simultaneously arrived to the country. The sources of this article are the official reports presented by the missions and the existing regulations supported by historiography, which allowed to determine the political and economic aspects of Colombia at different times. The Kemmerer (1923-1930), Currie (1949-1950), and Lebret (1954-1956) missions diagnosed the economic and social events of the country, and determined the conditions to undertake growth processes from the development of industrial technical education in its different levels, in order to comply with the requirements of external policies that promoted the development of Latin America, which encouraged the industrial technical training and the consolidation of the educational system in general.

\section{KEYWORDS:}

Industrial Development, Technical Education, International Economic Relations 



\section{Introducción}

El orden político soportado en un conflicto partidista; la violencia como forma para disipar las diferencias; la concentración de la mayor parte de la población en zonas rurales, donde carecen de servicios sanitarios y asistenciales; un alto porcentaje de analfabetismo; una fuerte influencia de la Iglesia católica en los asuntos públicos, y una economía inestable son, en parte, una expresión de Colombia durante la primera mitad del siglo XX. Sin embargo, desde sus primeras décadas, será clara la inclinación de asumir un proyecto de modernidad bajo parámetros internacionales, y abanderado por los gobernantes nacionales y locales; de allí la necesidad de concebir las condiciones políticas y económicas para el desarrollo de un capitalismo industrial que permita la consecución de un progreso técnico-material y social.

Desde inicios del siglo XX, en Colombia se presenta una serie de acontecimientos que llevan a forjar transformaciones en los diferentes ámbitos de la sociedad. Recursos como el petróleo, el café y el banano perfilan una economía agroexportadora; se dan acciones encaminadas a un desarrollo industrial, aumenta la inversión pública en obras de infraestructura y se incrementa la participación de los inversionistas extranjeros, capital imprescindible para jalonar los cambios que, a pesar de ser exiguos y tenues en algunos casos, exhiben la intención de iniciar procesos de modernización, propósitos compartidos con otros países latinoamericanos, pero con dinámicas particulares.

Aunado a lo anterior, sucesos de orden internacional, como las guerras mundiales, la potencialización económica de nuevos países y su política expansionista, repercutirán en el crecimiento e integración financiera de América Latina, aun cuando sea de forma paulatina y desigual. Colombia cuenta con una amplia disposición de materias primas, pero carece de mano de obra cualificada y de un desarrollo científico y tecnológico, aspectos que deberán corregirse para su incorporación a un sistema productivo mundial. Por esta razón, se presentan diversas estrategias económicas, entre ellas, la de modernizar los sistemas de producción y formar al personal con capacidad de asumir la transferencia tecnológica, tomando la educación especializada un lugar preponderante en las agendas gubernamentales.

Con el fin de subsanar las diferentes problemáticas presentadas y de incorporar al país a un proyecto de modernidad, se promueve el arribo de misiones económicas europeas y norteamericanas que, en el marco de modelos hegemónicos, darán recomendaciones de intervención política y financiera. La centralización del sistema educativo, su administración por parte del Estado, la incorporación de nuevas modalidades de formación, como la educación técnica industrial y la regulación legal de los niveles de enseñanza, son algunos de los cambios suscitados para modernizar 
los sistemas de producción y lograr un fortalecimiento financiero a partir de las recomendaciones efectuadas por las misiones económicas que asesoran a los diferentes gobiernos, aspectos que reafirman algunas de las propuestas realizadas por las misiones pedagógicas alemanas que, en varias oportunidades arriban, a Colombia.

Las misiones económicas diagnostican aspectos verificables en toda la población colombiana, y sus recomendaciones empiezan a ser puntos de referencia para la elaboración de planes quinquenales de desarrollo que, pese a concretarse con lentitud, orientan a un cierto adelanto económico y social, a partir de un cambio fundamental del sistema educativo. Se reconoce que, desde las últimas décadas del siglo XIX, los discursos del Gobierno nacional manifiestan la intención de generar cambios en los sistemas de producción orientados a la industria, los cuales demandan la formación de capital humano capacitado para impulsar los procesos de producción tecnificados. Si bien se concretan instituciones de educación para el desarrollo de las artes y los oficios, al igual que escuelas para la formación de ingenieros, en su mayoría son resultado de esfuerzos regionales, que con dificultad se harán extensivos en todo el país, dada la escasez de recursos de la nación y la falta de continuidad de las políticas del Gobierno central.

Las misiones económicas se convierten en referentes para evaluar y planear el sector educativo, se generan políticas de intervención estatal para favorecer las condiciones que posibilitan la ampliación de la cobertura escolar y la diversificación de la oferta desde los distintos niveles de formación que se reglamentan. Los informes de las misiones Kemmerer, Currie y Lebret, y otros documentos realizados por estos asesores extranjeros, al igual que artículos de economía e historia que analizan el trabajo de estas misiones, son las principales fuentes de información para la construcción de este texto. Este documento, a su vez, se nutre con documentos oficiales que notifican el accionar gubernamental y permiten establecer la transcendencia de las sugerencias dadas por las misiones para promover el desarrollo económico y el progreso social.

\section{Edwin Walter Kemmerer y el arribo de sus dos misiones (1923 y 1930)}

El economista norteamericano Edwin Walter Kemmerer participa en dos misiones contratadas en Colombia durante gobiernos políticamente diferentes, pero con la misma finalidad: potencializar la economía nacional. Kemmerer, especialista en teoría monetaria y finanzas públicas, orienta varias misiones en Latinoamérica. Su primer arribo a Colombia, para prestar sus servicios como asesor económico, se 
da en 1923, durante la presidencia del conservador Pedro Nel Ospina Vásquez. El propósito de esta misión de expertos es reestructurar el sistema financiero y fiscal, constituir instituciones que apoyen el crecimiento económico, y posicionar al país en la esfera internacional para facilitar la recepción de préstamos. Ospina Vásquez es ingeniero de minas de la Universidad de Berkeley y, como tal, vincula el progreso del país con la industrialización y la adecuación vial, procesos que demandan un vasto capital en un país sumido en la pobreza.

El estudio adelantado por la misión devela que la prosperidad del país está condicionada por la capacidad de atraer el capital extranjero, de allí que se presenta como eje estratégico la creación de un banco central que cuente con la participación de extranjeros en su junta administrativa. Asimismo, se recomienda la unificación y la conversión de la deuda externa; se convoca a la centralización, simplificación y ajuste del presupuesto oficial, y se sugiere la creación de la Contraloría General y la Superintendencia Bancaria (Kemmerer, 1927). De acuerdo con el estudio económico presentado por Jorge Valencia Restrepo (1987), el trabajo efectuado por la Misión Kemmerer y la influencia de este son determinantes para dar apertura a los títulos colombianos en el mercado neoyorquino. Estos logros, junto con las transformaciones institucionales recomendadas por la Misión Kemmerer y lideradas por Pedro Nel Ospina, favorecerán el crecimiento económico del país.

El efecto inmediato es el aumento de las exportaciones y del capital extranjero, a la par que se propician las condiciones para el surgimiento industrial. Se afirma que la economía colombiana experimenta, durante esta década, la mayor tasa de crecimiento de su historia (Meisel; Ramírez; Jaramillo, 2014). Además, se instauran el Ministerio de Hacienda y la Contraloría General de la Nación, para optimizar la recaudación de rentas, y la regulación y vigilancia del gasto público. La política económica y la inversión favorable para la construcción de obras públicas repercuten en el crecimiento industrial del país, de manera que se constituyen fábricas en regiones en las que, años atrás, eran inexistentes (Bejarano, 1980).

Aun cuando no son explícitas unas directrices en materia educativa, en el diagnóstico presentado por los expertos económicos es claro que un desarrollo económico apoyado en procesos de producción industrial demanda una mano de obra especializada. Para propiciar las transformaciones económicas, es perentoria la organización del sistema educativo, bajo una supervisión estatal, y la adopción de una participación donde la prestación del servicio esté a cargo del Gobierno, pues durante décadas la Iglesia católica es la que se encarga de esto. La Misión Kemmerer, con su balance económico, manifiesta la necesidad de una reestructuración educativa que permita disponer de una mano de obra cualificada; de allí el arribo de la Misión Pedagógica Alemana. 
Si bien, bajo la presidencia de José Manuel Marroquín, la Ley 39 de 1903 emite lineamientos sobre el sistema de instrucción pública, con los cuales el Estado asume mayor intervención en su organización, dirigencia y financiación, no es posible que en el plano práctico esto se traduzca en una ampliación del servicio educativo. La responsabilidad mayor de financiar los establecimientos de instrucción y garantizar las condiciones para su funcionamiento son asignadas a los gobiernos locales, muchos de ellos empobrecidos tras liderar guerras civiles, con condiciones poco favorables para concretar el proyecto de instrucción pública y lograr una política educativa nacional que supere iniciativas regionales.

De esta manera, a inicios de los años veinte continúa la necesidad de adoptar políticas públicas en educación, incluso que el ministerio a cargo asuma independencia del ramo de la salud; pero también de propiciar las condiciones para el cumplimiento de estas políticas. Algunos cambios empiezan a concretarse en 1927, como la supresión del Ministerio de Instrucción y Salubridad Pública, para dar paso al Ministerio de Educación Nacional. Si bien garantizar la educación pública a toda la población no se asume plenamente como responsabilidad del Estado, sí se obliga a los padres o tutores a proporcionar un mínimo de formación a los menores de edad. En cuanto a la educación secundaria, se regula el otorgamiento de títulos de bachilleres, facultando solo a las instituciones que cuenten con la aprobación del Ministerio de Educación Nacional; dicho título era condición necesaria para el ingreso a la educación universitaria (Ley 56, 1927). Aun cuando la financiación de los centros educativos oficiales continúa delegándose a los gobiernos departamentales, es importante la intención del Gobierno nacional de regular el sistema educativo e incentivar la educación elemental de la población.

Para 1930, el liberalismo político retoma el poder del país al conseguir en elección popular la presidencia de la República; en cabeza de Enrique Olaya Herrera se finaliza la hegemonía conservadora iniciada en 1886. Con la llegada del liberalismo, se acentúan las intenciones de propiciar grandes cambios que permitan la prosperidad económica y el desarrollo material y social. Es así como, en 1930, Edwin Walter Kemmerer retorna al país, contratado por el Gobierno nacional para brindar asesoría en manejo tributario, deuda externa, tasación del precio del café y la estabilización de las condiciones internas para enfrentar la crisis generada por la recesión económica mundial de 1929. A su vez, la misión tiene como objetivo reforzar la política de cooperación con Estados Unidos, pues es prioritario avivar la confianza entre los inversionistas y banqueros norteamericanos.

Tras dos meses de permanencia en Colombia, la misión de consejeros presenta, como resultado de su labor, 17 proyectos de ley, de los cuales 11 son puestos a consideración del Congreso, pero solo 7 logran convertirse en actos legislativos. Los 
proyectos que demandan mayor interés son los relacionados con el ajuste monetario, banca y política fiscal, con el fin de mantener un presupuesto equilibrado. El fortalecimiento de estos factores marca el punto de partida del desarrollo económico del país. Aunado al estudio de los recursos y prioridades sobre los cuales debía reposar el progreso nacional, es apremiante comenzar un proceso de industrialización, ampliar el mercado interno y capacitar al personal encargado de liderar los cambios y desempeñarse en las nuevas modalidades de trabajo que requieren los sectores de la producción que se están impulsando.

Las evaluaciones realizadas acentúan la necesidad de fortalecer una planeación económica sobre las bases de un conocimiento real del nivel de desarrollo de los diferentes sectores productivos y de las condiciones socioeconómicas de la población. Es así como, paulatinamente, se empiezan a conformar organismos públicos encargados de adelantar proyecciones en congruencia con el entorno nacional. A inicios de 1931, se instaura el Consejo de la Economía Nacional, cuyo propósito es favorecer la explotación de las riquezas naturales de Colombia, el despegue industrial, la organización bancaria y la vigilancia de las leyes que regulan la enseñanza agrícola, técnica industrial y comercial (Ley 23, 1931). Las responsabilidades asignadas al Consejo manifiestan la necesidad de intervenir en la educación y promover modalidades de formación que permitan la tecnificación de los procesos de producción, todo esto en el marco de una planeación económica.

De esta manera, potencializar la economía y hacerla sostenible con los recursos propios deberá ser una prioridad para el Gobierno nacional y, más allá de adoptar medidas políticas y económicas, se requiere la instauración de estrategias sociales que tiendan a garantizar un mejor nivel de vida, entre las cuales está la de brindar educación a la población. Un paso para ello es reglamentar la Ley 56 de 1927, mediante el Decreto 1790 de 1930, con el cual se interpone la obligatoriedad de enseñanza, se establece el pénsum de la formación primaria y se estipula que la edad de inicio escolar es a los 6 años. Por otra parte, para 1933, desde el Ministerio de Educación Nacional, se alienta a cursar estudios de educación superior como medio para liderar los procesos productivos del país (Carrizosa, 1933).

Desde finales del siglo XIX, está claro que el desarrollo económico del país demanda reformar las estructuras educativas; no obstante, las condiciones que trae consigo la nueva centuria y los balances dados por los analistas extranjeros que arriban al país con variados propósitos afianzan la necesidad de formular propuestas que precisen las dimensiones de cambio, en las que la relación entre educación y economía será cada vez más estrecha. Pronto pasará a ser insuficiente formar individuos con conocimientos básicos y bajo preceptos cristianos que calmen cualquier intención de sedición o de actuaciones que obstaculicen el progreso de la nación; se 
empieza a requerir la formación de personal cualificado para laborar en los diferentes modos de producción, a la par que se manifiesta la necesidad de educar en el país a quienes deban abanderar los procesos de modernización (Helg, 2001), pese a que esta posibilidad no está contemplada para todos los grupos sociales o, por lo menos, no en igualdad de condiciones. Las capas inferiores se forman en artes y oficios, los sectores medios se titulan como bachilleres técnicos industriales, agrícolas o comerciales, y una educación superior en ingeniería es para aquellos con mejores condiciones socioeconómicas.

Es preciso que el Gobierno adopte un mayor compromiso en la dirección, supervisión y financiación del servicio educativo, que se institucionalicen nuevas modalidades y niveles de enseñanza -entre ellas la educación técnica industrial-, que se cumpla la obligatoriedad de que todos los niños en edad escolar tengan un mínimo de educación, y que se modifiquen los métodos de enseñanza centrados en el aprendizaje memorístico y repetitivo. Sin embargo, la insuficiencia de fondos para invertir en nuevas escuelas o en la reestructuración de las existentes imposibilita dar cumplimiento a la enseñanza obligatoria durante los primeros años, problemática que debe esperar hasta los años cuarenta para empezar a solucionarse, a través de una mayor destinación de fondos para la construcción de edificaciones escolares (Decreto 1385, 1940). Otro aspecto significativo es la reglamentación de la segunda enseñanza o educación secundaria, la cual se enfoca en la formación técnica industrial, agrícola y comercial; para quienes no pueden ingresar a este nivel de enseñanza, se incentiva el acceso a las escuelas de artes y oficios (Melgarejo-Rey, 1931), instituciones de formación que, desde sus comienzos en las últimas décadas del siglo XIX, materializan las intenciones de escolarizar y tecnificar los trabajos manuales, con los cuales se generan transformaciones en los procesos productivos.

Con el liberalismo en el poder, se reafirma el propósito de transitar hacia una sociedad urbana e industrial, así como de tecnificar los procesos de producción y modernizar las diferentes estructuras económicas, por lo que la consolidación de una educación técnica es un asunto prioritario (Díaz; León, 2008); de allí la instauración de una política de instrucción técnica y de un marco legal que la avale. Es notoria la influencia de una política nacional con el propósito de fortalecer el desarrollo económico, con base en la producción industrial y en un sistema educativo a través de escuelas de artes y oficios, institutos industriales que oferten el bachillerato técnico y de facultades técnicas que formen ingenieros, en especial, constituidas en las capitales de los departamentos que vislumbran las condiciones para el despegue del sector industrial, como lo son Bogotá y Medellín, o en algunas ciudades con escenarios poco favorables y trascendencia menor, como Bucaramanga. Con el ánimo de integrar los diferentes niveles de formación, la culminación del bachillerato técnico asegura la 
admisión en las facultades o universidades técnicas y, posteriormente, se estipula la integración institucional para la formación de cuadros completos de técnicos.

Con la llegada a la presidencia del liberal Alfonso López Pumarejo y su política de Revolución en Marcha (1934-1938), se acoge con mayor fuerza la reforma educativa, cuyos propósitos se centran en mejorar y ampliar la prestación del servicio educativo, a la par que se busca tecnificar la mano de obra para laborar en las industrias y en el campo y, con ello, afianzar las exportaciones y generar mayores ingresos que permitan concretar el proyecto de modernización. De esta manera, se centralizan los planes de estudio y el Estado intenta asumir la intervención plena en el sistema educativo, papel que, hasta el momento, había cumplido la Iglesia (Castro, 1938).

El gobierno liberal y los que le siguen son claros en sus propósitos de reestructurar el sistema educativo y apersonarse de su dirección, limitando las atribuciones de la Iglesia católica en la administración y orientación de la instrucción pública, concedidas por la Constitución de 1886 y el Concordato de 1887. En esta línea de intervención educativa, se incrementa por cuatro el presupuesto nacional destinado a este sector, aun cuando es insuficiente para la ejecución de grandes proyectos y las arcas departamentales o municipales continúan soportando el mayor porcentaje de financiación (Helg, 2001). La política educativa liberal se refleja en una centralización de la instrucción pública, en la estandarización de los programas y en un mayor control y vigilancia del acto educativo que, aunado a mayores partidas presupuestarias, permite que proyectos regionales de educación técnica encuentren respaldo para su fomento.

Ejemplo de lo anterior es la Escuela de Artes y Oficios de Santander, ubicada en su capital. Aun cuando recibe gran acogida en la población, y en ella se centran esperanzas de mejora social y económica, la iliquidez del gobierno y los conflictos civiles en los que participa su población determinan un funcionamiento intermitente hasta llevar a su cierre; con dinámicas de reapertura y clausura hasta los años treinta, cuando las nuevas directrices de educación nacional y las condiciones de desarrollo local orientan su función a institución industrial.

La Ley 12 de 1934 promueve la enseñanza obrera mediante la fundación y organización de las escuelas complementarias de oficios, para la cualificación de la mano de obra. Esta iniciativa se enmarca en lo que se denomina Campaña de Cultura Aldeana y Rural, la cual busca reorganizar el Ministerio de Educación Nacional, estimular la apertura de escuelas normales, afianzar la educación rural y regular la prestación del servicio educativo desde los diferentes niveles, obligando a los establecimientos de formación a cumplir con un mínimo de requisitos para continuar funcionando o recibiendo financiamiento cuando sean del Estado. Asimismo, se compromete a invertir en educación un $10 \%$ del presupuesto general, a partir de 1936. 
Dada la homogeneidad de la formación impartida en el bachillerato y las críticas que desde el mismo Gobierno se emiten por la poca utilidad que seis años de formación secundaria dan a las personas con relación a los requerimientos de su entorno (Gaitán, 1940), es necesario establecer los planes de estudio de este nivel de formación, para equilibrar la cantidad de asignaturas que deben cursarse y su intensidad horaria, a la par que se fragmenta la finalidad de la formación. En consecuencia, el bachillerato se diversifica para darle relevancia a la orientación técnica, además de permitirle a los colegios tener libertad para intensificar las áreas que respondieran a las tradiciones y necesidades de cada región o a las exigencias de cada sexo (Decreto 1570, 1939). La expedición de normativa, como el Decreto 1972 de 1933, consiente el ingreso de mujeres a las facultades universitarias y, con ello, su integración a los sectores productivos.

Con las mismas intenciones, a finales de 1939, se reglamenta la enseñanza industrial no vinculada a la educación formal (Decreto 2350, 1939); sin embargo, su curso se condiciona a la finalización de los cuatro años de la enseñanza primaria. Su propósito es preparar al personal de operarios y técnicos en las diversas especialidades que demanda la industria y en las necesidades del país, mientras que su organización y vigilancia se encomienda a la Sección de Enseñanza Industrial y Complementaria del Ministerio de Educación Nacional.

Antes de culminar el segundo año de haber sido expedida la anterior disposición, es complementada con el Decreto 1593 de 1941, el cual reitera la necesidad inmediata de preparar a la juventud para el trabajo industrial en sus diferentes niveles y modalidades, por lo que se procede a instaurar el Consejo Nacional de Enseñanza Industrial y Complementaria, integrado, en primera instancia, por los ministros de Educación y de Economía, lo que reafirma que las políticas educativas son pensadas en respuesta a un proyecto económico.

Si bien en los años treinta se afianza la relación entre educación y desarrollo, es a partir de la década siguiente que el proyecto logra formalizarse con notorios despegues y un fuerte impulso a la formación vocacional; se insiste en la necesidad de instruir a la población para el ejercicio práctico de ciertas ciencias, artes y oficios. Jorge Eliécer Gaitán, como ministro de Educación (1940-1941), plantea que la preparación técnica de los trabajadores colombianos está en desacuerdo con el desarrollo económico e industrial, por lo que debía acudirse a la contratación de expertos extranjeros; de allí la insistencia en reorganizar el sistema educativo y la oferta de programas técnicos industriales (Gaitán, 1940).

En la Asamblea de Directores de Educación que se realizó en 1940, en Bogotá, se conciertan las directrices para la implementación del bachillerato industrial y se concluye que una de las formas de impulsar la industria nacional es formar cuadros 
de técnicos, es decir, la formación consecutiva de todos los niveles de educación técnica: obreros auxiliares, obreros calificados, expertos, técnicos e ingenieros. La constitución del bachillerato técnico se justifica en la importancia de "brindar a las personas una educación que permitiera contar con un conocimiento para realizar una labor útil en el terreno científico, un conocimiento general de las bases de la educación técnica y el dominio de algún oficio especial que les facilitara ocupar un puesto en el amplio mundo de la producción" (Galán, 2011:59). Por su parte, con la formación en ingeniería se busca "hermanar la ciencia con la técnica" (Galán, 2011: 59).

Con frecuencia, se encuentra en las disposiciones gubernamentales la necesidad de impulsar la formación técnica industrial desde el nivel superior, ampliando el número de centros universitarios que oferten este tipo de educación. Vale reconocer que durante la segunda mitad del siglo XIX se fundan la Universidad Nacional y la Escuela de Minas de Medellín, establecimientos importantes para la profesionalización de la formación técnica industrial, pero insuficientes para formar la fuerza laboral que demanda la transformación de los procesos productivos bajo una nueva racionalidad económica. En coherencia con estas intenciones, se inauguran la Universidad del Atlántico (1941), la Universidad de Caldas (1943), la Universidad del Valle (1945) y, 3 años después, se constituye la Universidad Industrial de Santander.

En síntesis, las misiones Kemmerer evalúan las condiciones económicas de Colombia y plantean estrategias de intervención que alientan el interés en generar procesos de modernización, centrados en la promoción de sistemas industrializados de producción, para lo cual es perentorio atenuar los problemas educativos de la población y la institucionalización de una política educativa que permita, desde los diferentes niveles y modalidades formativas, contar con mano de obra calificada. Estas misiones acentúan la necesidad de una participación estatal enfocada en la orientación de la instrucción pública y en la consecución de los proyectos educativos, acciones que empiezan a percibirse desde los actos legislativos, aun cuando muchos no logran trascender el plano formal.

\section{Lauchlin Currie y su programa de desarrollo para el progreso (1949-1950)}

A finales de 1945, se oficializa la conformación de la Organización de Naciones Unidas (ONU), con el ánimo de garantizar seguridad, propiciar las relaciones de paz y ayudar a superar situaciones adversas como la pobreza, las enfermedades y el analfabetismo, entre otros factores que impiden el desarrollo económico y el progreso social. Dependiente de la ONU, se crea en 1948 la Comisión Económica 
para América Latina y el Caribe (CEPAL); su fin se centra en apoyar a los gobiernos latinoamericanos en el desarrollo económico, fortaleciendo las relaciones comerciales y propiciando las condiciones para mejorar el nivel de los pueblos. Asimismo, unos años antes se ha instaurado la Organización de las Naciones Unidas para la Educación, la Ciencia y la Cultura (Unesco), cuyo propósito es la búsqueda de la paz y la seguridad mediante la formación intelectual y moral de la población.

De esta manera, la educación toma un papel relevante en los programas de gobierno y se afirma la relación previamente establecida entre el desarrollo social y el económico. Dadas las condiciones que presentaba el panorama internacional, el Gobierno colombiano, presidido por el conservador Mariano Ospina Pérez, considera que es el momento oportuno para impulsar la economía nacional y, a mediados de 1948, presenta ante el Banco Mundial una solicitud de préstamo para financiar la construcción de carreteras, ferrocarriles, plantas hidroeléctricas y un puerto. Desde las altas directivas del Banco Mundial, se condiciona cualquier ayuda al envío de una misión para determinar las necesidades más apremiantes del país mediante un estudio exhaustivo del panorama económico nacional, cuyo propósito debe acentuarse en la elevación del nivel de vida del pueblo colombiano (Sáenz, 2001).

Desde julio hasta diciembre de 1949, la primera misión organizada por el Banco Mundial trabaja en Colombia, bajo la dirección del economista canadiense Lauchlin Currie, quien ha seleccionado a un grupo de economistas norteamericanos y de funcionarios de ese Gobierno. Entre las fortalezas del equipo de trabajo se reconoce su diversidad profesional, que incluye desde expertos en finanzas públicas hasta médicos, permitiendo un análisis variado y detallado de las condiciones en las que se encuentra el país, con el cual se pueden generar propuestas más acertadas para mejorar (Currie, 1993).

Posteriormente, en la II Reunión de Ministros de Educación, celebrada en la ciudad de Lima durante mayo de 1955, Colombia será presentada como uno de los primeros Estados latinoamericanos en planificar su desarrollo, destacándose el avance dado en la proyección educativa dentro de un marco general de desarrollo económico. En este evento se reconoce que la llegada de las misiones económicas a algunos países latinoamericanos promueve de manera temprana el adelanto de experiencias de planificación educativa (Desarrollo del proyecto, 1960).

La estrategia de desarrollo planteada por la Misión Currie centra su atención, primero, en lo referente al sistema económico, en especial lo relacionado con la inflación y el transporte; y luego se enfoca en la salud, la higiene, la educación y los servicios públicos. De esta manera, la propuesta de este economista se enfoca en reorganizar los recursos existentes, introducir y hacer extensivas nuevas técnicas de producción y direccionar el capital hacia canales más productivos y socialmente 
más benéficos (Banco Mundial, 1950). Teniendo en cuenta que el sector agrícola colombiano provee una diversidad de alimentos, fibras y productos de exportación, se deben buscar alternativas para aumentar su productividad, como la optimización de la fuerza laboral, porque se podría contar con una mayor mano de obra para la producción de otros elementos. La misión es partidaria de promover las migraciones a los centros urbanos para disponer de la mano de obra suficiente y, en lo posible, capacitada en el trabajo fabril, en el área de la construcción, transporte, servicios públicos o educación (Banco Mundial, 1950).

En lo concerniente a la educación, los índices de analfabetismo van en aumento y superan el $40 \%$ de la población. Por si fuera poco, solo la mitad de los niños entre 7 y 11 años recibe formación primaria y, de ellos, un número reducido logra concluir este nivel de formación. Las escuelas de artes y oficios y las escuelas industriales prestan el servicio educativo a 4000 estudiantes en todo el país; las agrícolas vocacionales, a 800 y, con poca precisión, se expresa que un mínimo de la población logra ser bachiller. Estas condiciones precarias necesitan superarse para elevar el nivel de vida del pueblo colombiano, mejorar el capital humano y centrar las bases del progreso económico y social (Banco Mundial, 1950).

La Misión Currie, en Colombia, pronuncia un discurso hegemónico de desarrollo que demanda la modernización de los obsoletos sistemas de producción y la formación de individuos con capacidad de afrontar el proceso de transferencia tecnológica; la salida de este panorama será invertir en educación. Precisamente, las recomendaciones del Banco Mundial se orientan hacia la incorporación del país al sistema productivo mundial mediante inversión en educación, especialmente técnica. De acuerdo con el informe, es prioritario instaurar una política real de capacitación de la mano de obra, que contribuya a incrementar la productividad de las industrias y, por ende, el margen de utilidades. Para ello, será prioritario empezar a vincular la educación técnica con la industria; se recomienda que industriales se comprometan en el otorgamiento de fondos y servicios para la capacitación de sus trabajadores o de aquellos que vayan a ingresar. Asimismo, se recuerda la necesidad de consolidar un bachillerato que capacite para el trabajo productivo, pues este debe dejar de ser solo una preparación para ingresar a la universidad.

Desde finales de 1948, la Ley 143 define la educación técnica como el constituyente práctico de la educación orientada al trabajo racionalizado y se organiza por secciones: industriales, agrícolas, comerciales y de economía doméstica; asimismo, estandariza planes y programas por niveles educativos. Estos cambios permitirán contar con cuadros completos de técnicos, partiendo del auxiliar hasta llegar al ingeniero. Dos años antes, se ha decretado la conformación del bachillerato técnico industrial. Los estudiantes que cursen los primeros cuatro años se titularán como 
expertos industriales, y aquellos que concluyan los seis años de estudio serán bachilleres técnicos industriales y se les permitirá ingresar, sin objeción, a las Facultades de Ingeniería (Decreto 884, 1946).

Para impulsar la tecnificación de los procesos de producción y el comercio a gran escala, se adopta una serie de políticas económicas y sociales, en coherencia con un proyecto de modernización. Para este propósito, se conforman los comités de acción, para impulsar el desarrollo comercial e industrial de las distintas regiones. En el caso específico del comité de acción del departamento de Santander, más allá de sus funciones económicas, se gestiona la fundación de una universidad industrial, con el argumento de constituir un sistema educativo capaz de responder a las exigencias de un modelo acentuado en la industrialización (Galán, 2008).

Es importante reconocer las iniciativas del Gobierno colombiano y de los distintos sectores económicos por promover una educación industrial. Por esta razón, desde los años treinta se dan avances en su reglamentación y en la generación de condiciones para la prestación de su servicio. No obstante, a mediados del siglo XX es aún muy cuestionada la formación que imparten algunos de los establecimientos destinados a la enseñanza de la educación técnica; se llega a decir que no se encuentran a la vanguardia de los cambios técnicos en la producción, por lo que con dificultad pueden responder a los procesos de industrialización nacional. Si bien existe una preferencia por el bachillerato clásico, los pocos que alcanzan un bachillerato técnico en contadas ocasiones pueden adelantar estudios de nivel superior en estas áreas, dado el escaso número de universidades con orientación industrial, inconvenientes que llevan al Gobierno nacional y a la Asociación Nacional de Empresarios de Colombia a buscar alternativas para dar continuidad a los estudios técnicos superiores, aun cuando esto implique financiar la formación en el extranjero.

Tras la entrega del informe realizado por la Misión Currie y, en especial, su llamado a la vinculación de los esfuerzos del Estado y de los sectores productivos, es evidente el interés de estos por modificar las condiciones adversas que limitan el desarrollo nacional o que impiden recibir ayudas internacionales. En 1950, se crea el Instituto Colombiano de Especialización Técnica en el Exterior (Icetex), anexo al Ministerio de Educación Nacional (Decreto 2586, 1950); su constitución obedece a la necesidad de contar con capital humano cualificado para adelantar procesos de producción tecnificados y aprovechar las riquezas naturales con las que cuenta el país. Por último, la creación del Icetex se justifica en la necesidad de generar posibilidades para que la clase media, los campesinos y los obreros puedan adelantar especializaciones técnicas fuera del país. Con la creación de esta institución de promoción y financiación de la educación técnica, el Gobierno colombiano busca ajustarse a los lineamientos dados por el Banco Mundial con el fin de disponer de 
la mano de obra especializada para la transferencia tecnológica y el desarrollo de los procesos de producción industriales.

En definitiva, la Misión Currie es clara al expresar la necesidad de incorporar al país al sistema económico mundial, para lo cual prioriza políticas públicas que incrementan la inversión en educación. Desde marcos hegemónicos de desarrollo, se insta a la modernización de los procesos productivos y, con ello, a la formación del personal capaz de asumir la transferencia tecnológica, conduciendo a experiencias de planeación educativa que más adelante son reconocidas en escenarios internacionales.

\section{Louis-Joseph Lebret: economía y humanismo (1954-1957)}

Ni los gobiernos liberales ni los conservadores, y menos el del general Gustavo Rojas Pinilla (1953-1957), serán ajenos al arribo de misiones. Los informes de las misiones ofrecen garantías a los inversionistas extranjeros y dan garantías a los gobiernos nacionales para implementar políticas públicas sobre la base de diagnósticos más detallados sobre las condiciones del país. Con la llegada de cada misión, se evidencia aún más la necesidad de realizar un estudio de las condiciones sociales de la población colombiana para planear el desarrollo económico, razón por la cual la misión de 1954 se enmarca en el trabajo de economía y humanismo, presidido por Louis-Joseph Lebret.

Lebret hace su arribo a Colombia en 1953, pero solo un año más tarde acuerda con el presidente Rojas Pinilla realizar un estudio de las condiciones de desarrollo del país. La Misión de Economía y Humanismo efectúa su estudio en el país entre diciembre de 1954 y julio de 1956. Dos años más tarde, para 1958, la misión entrega su informe bajo el nombre de Estudio sobre las condiciones del desarrollo de Colombia. Durante más de un año, la misión recorre 45 municipios y 11 ciudades del territorio colombiano. Se busca tener un mayor acercamiento con los sectores populares, e información de lugares apartados compilada por expertos y organismos oficiales, con el fin de dar a conocer, en lo posible, las particularidades del país para evitar brindar recomendaciones que no se ajusten a la realidad nacional, dado que, aun cuando las necesidades sean las mismas para algunos países o regiones, las posibilidades de satisfacerlas pueden ser diferentes (Lebret, 1958).

Concluida su labor, Lebret y el Centro de Economía y Humanismo deben entregar el informe de su trabajo a un Gobierno distinto al que los contrató. Tras el derrocamiento de Rojas Pinilla y la adjudicación del poder, por parte de este, a la Junta Militar, deviene un gobierno de concertación bipartidista, cuyo primer presidente es Alberto Lleras Camargo. Más allá de presentar en detalle una radiografía del 
país, el informe ofrece una vasta lista de las necesidades o problemáticas que urgen atención inmediata. Cabe enfatizar que el informe de la misión direcciona todas sus recomendaciones a reducir la brecha social. Se destaca que otro problema es la poca titulación para el trabajo, en especial en el área técnica, factor que obstruye el desarrollo de nuevos procesos de producción industrial y agrícola (Lebret, 1958).

De esta manera, la educación de la población también ocupa la atención de la misión y es el eje temático abordado en la última parte del informe. Al respecto, se expresa que el acceso al sistema educativo es desigual, por lo que se reitera la urgencia de garantizar la instrucción básica a toda la población y generar las condiciones para ampliar el ingreso a la educación superior, condicionado a los méritos. El acercamiento directo a las diferentes problemáticas que integran la realidad colombiana y la misma filosofía humanista que enmarca la misión hacen que se priorice el desarrollo social ante la necesidad de alcanzar el crecimiento económico, razón por la cual la educación de la población es una prioridad (Lebret, 1958).

Las propuestas de esta misión se enfocan más en resaltar las bondades de la educación que en expresar una ruta a seguir. Se insiste en que la promoción de las industrias y la tecnificación de los procesos agrícolas demandan de un personal formado para ello. Lebret y su equipo de trabajo alertan sobre la deficiente enseñanza industrial: existe una carencia de centros artesanales para capacitar obreros y de instituciones industriales que formen en técnicas modernas. Reiteran lo ya expuesto por la Misión Currie acerca del compromiso que deben asumir las diferentes agremiaciones productivas en la cualificación de sus trabajadores. Abiertamente, se hace un llamado a racionalizar el trabajo y el capital, especialmente la fuerza laboral, para alcanzar mayor productividad y posicionamiento a escala internacional. En términos generales, la educación es percibida como un factor de cambio y una condición para alcanzar una nueva etapa de desarrollo, aspecto que, desde años atrás, se percibe como prioridad.

Si bien la Misión Lebret logra publicar su informe en 1958, algunos pormenores del trabajo son entregados previamente al Gobierno nacional y sirven como base del Primer Plan Quinquenal de Colombia para la Educación (Helg, 2001). Desde 1955, el ministro de Educación Nacional, Gabriel Betancur Mejía, propone la elaboración de una planeación integral de la educación en Colombia, apoyada en los expertos extranjeros y nacionales que adelantan trabajos de investigación en este campo (Sánchez, 1967). Tras las reiteradas sugerencias de los asesores económicos y las directrices dadas en eventos educativos, como la II Reunión de Ministros de Educación auspiciada por la Unesco, se insiste en la necesidad de constituir organismos específicos de planeación educativa que apoyen al ministerio encargado de este ramo (Desarrollo del proyecto, 1960). 
De esta manera, en 1957, se instituye la Oficina de Planeamiento Educativo. Al momento de oficializar su apertura, quienes están a cargo manifiestan que este organismo suplirá la carencia de una verdadera administración pública de la educación, y entre sus responsabilidades iniciales está la elaboración del plan quinquenal, el cual establece, como primera tarea, determinar con claridad las necesidades económicas de la educación para analizar las posibles alternativas de solución (República de Colombia, 1957).

Entre las principales propuestas contenidas en este plan está la racionalización del uso de los recursos, la democratización de las oportunidades de estudio y la modernización de la concepción de la educación frente al desarrollo económico y social; también la reforma al sistema de educación primaria y media, la formación técnica, la educación normalista y la creación de un servicio nacional de aprendizaje, según recomendaciones de la Misión Lebret. Igualmente, se hace un llamado a afianzar los vínculos entre el ministro de Educación, el ministro de Trabajo y Fomento, y el director del Comité de Planeación, para promover una formación que responda a los requerimientos del país.

Dos aspectos que merecen mencionarse por ser importantes para el desarrollo de la educación técnica en el área industrial, y por ser el resultado del estudio presentado por Lebret y afianzado por el plan quinquenal, son la reglamentación para fortalecer el bachillerato técnico y la creación del Servicio Nacional de Aprendizaje (SENA). A través del Decreto 884 de 1946, se crea el bachillerato técnico, pero ante la premura de contar con el personal capacitado para responder a las necesidades de la industria y el comercio, en 1959 el Gobierno nacional considera conveniente reestructurar sus planes de estudio y los requisitos para alcanzar su titulación. Considerando las recomendaciones de Lebret, se propone integrar esta formación con el campo laboral práctico, por lo que se decreta que la formación técnica industrial pasará de seis a siete años; cursados y aprobados los primeros cuatro se otorgará el título de experto, para lo cual es requisito acreditar un año de experiencia en una empresa, demostrando suficiencia en la especialidad seleccionada (Decreto 2433, 1959).

El 10 de mayo de 1957, sale de la presidencia de la República el general Gustavo Rojas Pinilla, y el poder es asumido por una Junta Militar, la cual gobernará hasta 1958, cuando entrega la administración del país a una alianza bipartidista. En medio de la difícil situación de orden público, la Junta Militar debe continuar con el proyecto de modernización nacional, adecuando las condiciones del país para el desarrollo económico y social, y acatando las recomendaciones de los expertos extranjeros. Es así como se decreta la creación del SENA, sustentada en el deber que tiene el Gobierno de propugnar por la enseñanza técnica de las clases trabajadoras y en 
la obligatoriedad de atender las necesidades de las clases menos favorecidas para fomentar su mejoramiento (Decreto 118, 1957).

El SENA se enfoca en brindar formación profesional a los trabajadores de la industria, el comercio, la agricultura, la ganadería y la minería. Además de la preparación técnica del trabajador, se orienta para la formación de ciudadanos socialmente útiles y responsables que contribuyan al crecimiento económico y al mantenimiento de la armonía social. Asimismo, a esta institución se le atribuyen las funciones de contribuir con los patronos y trabajadores para constituir un sistema nacional de aprendizaje, organizar y supervisar en todo el país la enseñanza teórica y práctica de los oficios u ocupaciones que exijan formación profesional metódica, organizar cursos complementarios de preparación para los trabajadores técnicos y administrativos de todos los niveles, y contribuir con el desarrollo de investigaciones relacionadas con la organización científica del trabajo (Decreto 164, 1957).

La dirección superior del SENA es asignada a un consejo nacional, integrado por representantes del Ministerio del Trabajo, del Ministerio de Educación Nacional, de la Federación Nacional de Comerciantes (Fenalco), de la Asociación Nacional de Industriales (ANDI), de los agricultores y ganaderos, de los trabajadores y de la Iglesia católica, en cabeza del arzobispo primado de Colombia. Como lo sugieren los expertos extranjeros, se dispone la colaboración de todos los estamentos políticos y económicos para fortalecer la cualificación del trabajador, vinculando, de manera racional y técnica, sus esfuerzos al crecimiento económico y a una elevación de su nivel de vida.

A manera de conclusión, la Misión Lebret acentúa el trabajo de las misiones que la antecedieron, retrata el duro panorama social y ofrece recomendaciones económicas que alcanzan una mayor puesta en práctica; la perentoria incorporación al mercado mundial y las contenidas disputas partidistas tras la coalición política de 1958 facilitan la ejecución de políticas públicas para el desarrollo socioeconómico. En el ámbito educativo, se reconoce su planeación y vinculación en el marco de un proyecto de modernización nacional; se instaura la Oficina de Planeación Educativa, articulada al Ministerio de Educación y a las disposiciones de organismos internacionales como la Unesco. Por su parte, la educación técnica continúa siendo asumida como condición sustancial para el ascenso de la industria, priorizándose las condiciones para su promoción desde los distintos niveles de formación. 


\section{Conclusiones}

Se puede considerar, acatando las recomendaciones de las misiones económicas para potencializar la agricultura y la naciente industria en Colombia, que es imperioso para los gobiernos del momento implementar reformas educativas. Es claro que los lineamientos son cumplidos hasta donde las condiciones propias del país lo permiten. Sin distinción política, gobiernos conservadores y liberales acuden a la asesoría de expertos norteamericanos, como Edwin Kemmerer, que pese a sugerir políticas estandarizadas para América Latina, y ser más de orden económico que social, incentivan al Gobierno nacional a emprender diversos procesos de reestructuración, aun cuando solo sea para demostrar que se cumplen los requerimientos de la banca y del Gobierno de Estados Unidos.

Si bien se avanza en la institucionalización de entidades para la organización, control y promoción del sistema educativo nacional, al igual que en la ampliación de la oferta educativa y en los establecimientos de formación, el presupuesto para la educación siempre será inferior a los requerimientos de la nación y a las expectativas del Gobierno, en especial porque la mayoría de las veces son las administraciones departamentales y municipales, o las comunidades religiosas quienes tengan que abanderar los proyectos educativos y sufragar sus gastos.

Durante los años cincuenta del siglo XX, se acentúa la concepción de supeditar el progreso material al desarrollo de las fuerzas productivas y a la adopción del conocimiento científico y tecnológico. Para alcanzar este objetivo, es apremiante reestructurar y reglamentar el sistema educativo, mediante la incorporación de nuevas modalidades de formación para el trabajo, en las que se impartan conocimientos más complejos y una marcada orientación tecnológica.

Como criterio de orden mundial, se acuerda que la elevación del nivel de vida de la población requiere la planificación de la educación, al mismo tiempo que la de la economía; de manera que la llegada de las misiones a los países latinoamericanos promueve, de forma temprana, el adelanto de experiencias de programación educativa. En Colombia, se evidencia la necesidad de enmarcar la economía en un riguroso planeamiento, con el cual soslayar la improvisación y dispersión de los recursos para favorecer el crecimiento nacional. La asesoría y apoyo financiero de entidades internacionales acentúa, en el Gobierno colombiano, el propósito de organizar la función pública como requerimiento para alcanzar mayor eficiencia de los procesos económicos, aspecto que propicia la constitución de una política social orientada a fortalecer el sistema de educación y de salud, así como la ejecución de obras públicas.

Sin desconocer los adelantos en el ámbito educativo alcanzados desde finales del siglo XIX ni las condiciones particulares gestadas en el contexto nacional, se debe 
destacar el aporte de las misiones económicas en la instauración de una política de promoción de la educación primaria, básica y media, así como el impulso dado a la instrucción técnica desde los diferentes niveles de formación. Vale recordar que, contrario a las misiones pedagógicas -en especial la alemana- que arriban al país en diferentes momentos, las misiones económicas no aportan lineamientos en lo concerniente a la didáctica y pedagogía de los procesos formativos, sino que su contribución recae más en subrayar la necesidad de adecuar el sistema educativo para propiciar el desarrollo de un capitalismo sustentado en la tecnificación de los procesos, en encauzar las políticas públicas a la planeación de un proyecto de modernidad enmarcado en parámetros de orden internacional, abanderado por las élites locales, pero supeditado a las especialidades del contexto.

Los expertos que integran las misiones y los organismos internacionales que están detrás de estos se convierten en nuevos referentes discursivos para planear un proyecto de modernidad, ajustado a las condiciones del contexto nacional en ocasiones adversas. Las experiencias locales en educación, dadas desde finales del siglo XIX y abanderadas por comunidades religiosas y por personalidades con amplio reconocimiento e influencia regional, inician procesos de reestructuración para ajustarse a una política educativa nacional diseñada de acuerdo con un modelo internacional, cuyo éxito o fracaso está condicionado por las circunstancias regionales en las que emergen.

En términos generales, al rastrear la labor de algunas misiones económicas que arriban a Colombia durante el siglo XX, se puede plantear que, tras sus recomendaciones para promover el desarrollo económico y mejorar el nivel de vida de la población, la planificación de la educación se constituye como una prioridad que debe reflejarse en los planes generales de desarrollo; los gastos de funcionamiento del sistema educativo requieren concebirse como inversión para el crecimiento financiero y se debe promover con mayor medida la educación técnica en todos sus niveles de formación. De allí que, desde los años cincuenta, predominan los estudios y planes sobre el papel económico de la educación; países en los diferentes continentes empiezan a constituir planes de educación vinculados a sus planes de desarrollo económico y social, y a generar condiciones reales para su implementación. La prioridad educativa se centra en contar con mano de obra cualificada y un proyecto que potencialice los diferentes sectores productivos. 


\section{Referencias}

Banco Mundial (1950). Bases de un programa de fomento para Colombia. Informe de una misión dirigida por Lauchlin Currie. Bogotá: Banco de la República.

Bejarano, Jesús (1980). La economía en el siglo XX. En Manual de Historia de Colombia (Tomo III, pp. 17-79), editado por Jaime Jaramillo-Uribe. Bogotá: Colcultura.

Carrizosa, Julio (1933). Memoria del ministro de Educación Nacional al Congreso. Bogotá: Cromos.

Castro, José Joaquín (ed.), (1938). Educación Nacional: Informe al Congreso. Bogotá: ABC.

Currie, Lauchlin (1993). Los objetivos del desarrollo. Cuadernos de Economía, 13(18-19), 163-188.

Decreto 1570 de 1939 (2 de agosto), por el cual se fija el plan de estudios de educación secundaria. Diario Oficial núm. 24140. Recuperado en https://www.mineducacion.gov. co/1759/articles-102775_archivo_pdf.pdf

Decreto 2350 de 1939 (7 de diciembre), por el cual se reglamenta la enseñanza industrial. Diario Oficial núm. 24239. Recuperado de http://www.suin-juriscol.gov.co/viewDocument. asp?ruta=Decretos $/ 1439958$

Decreto 1385 de 1940 (17 de julio), por lo cual se reglamenta el Decreto 503 de 1940, en lo relativo a construcciones escolares. Diario Oficial núm. 24420. Recuperado de http://www. suin-juriscol.gov.co/viewDocument.asp?id=1277161

Decreto 884 de 1946 (16 de marzo), por el cual se crea el Bachillerato Técnico Industrial y se fija el correspondiente plan de estudios. Diario Oficial núm. 26093. Recuperado de http:// www.suin-juriscol.gov.co/viewDocument.asp?ruta=Decretos/1183794

Decreto 2586 de 1950 (6 de agosto), por el cual se crea el Instituto Colombiano de Especialización Técnica en el Exterior. Diario Oficial núm.27383. Recuperado de https://www.mineducacion. gov.co/1759/articles-103405_archivo_pdf.pdf

Decreto 118 de 1957 (21 de junio), por el cual se decretan aumentos de salarios, se establece el subsidio familiar y se crea el Servicio Nacional de Aprendizaje, SENA. Diario Oficial núm. 29441. Recuperado de http://www2.congreso.gob.pe/sicr/cendocbib/con4_uibd. nsf/38B359B895555A6905257FE20078E2A4/\$FILE/1.DECRETO_118_DE_1957_crea.pdf

Decreto 164 de 1957 (6 de agosto), por el cual se organiza el Servicio Nacional de Aprendizaje. Diario Oficial núm. 29475. Recuperado en https://www.redjurista.com/Documents/ decreto_164_de_1957_poder_ejecutivo.aspx\#/

Decreto 2433 de 1959 (11 de septiembre), por el cual se modifican los planes de estudios para la enseñanza industrial y comercial. Diario Oficial núm.30076. Recuperado de https://www. mineducacion.gov.co/1759/articles-10360o_archivo_pdf.pdf 
Desarrollo del proyecto principal de la Unesco sobre educación primaria en Iberoamérica (1960). Revista de Educación, 39(112), 35-39.

Díaz, Ariel; León, Libardo (2008). Historia de una universidad del medio siglo: la Universidad Industrial de Santander. Bucaramanga: UIS.

Gaitán, Jorge Eliécer (1940). Obra educativa del Gobierno en 1940. Bogotá: Imprenta Nacional.

Galán, Mario (2008). Una obra vital para Santander. Revista de Santander, 3, 18-21.

Galán, Gabriel (2011). Mario Galán Gómez: un hombre hecho por sí mismo. Bucaramanga: Universidad Industrial de Santander.

Helg, Aline (2001). La educación en Colombia 1918-1957. Bogotá: Universidad Pedagógica Nacional.

Kemmerer, Edwin (1927). Economic advisory work for governments. The American Economic Review, 17(1), 1-12.

Lebret, Louis-Joseph (1958). Estudio sobre las condiciones del desarrollo de Colombia. Bogotá: Cromos.

Ley 56 de 1927 (10 de noviembre), por el cual se dictan algunas disposiciones sobre instrucción pública.Diario Oficialnúm. 20645. Recuperado de https://www.mineducacion.gov.co/1621/ articles-102998_archivo_pdf.pdf

Ley 23 de 1931 (9 de febrero), por el cual se crea un Consejo de Economía Nacional. Diario Oficial núm. 21619. Recuperado de http://www.suin-juriscol.gov.co/viewDocument. asp?ruta $=$ Leyes $/ 1578740$

Meisel, Adolfo; Ramírez, María Teresa; Jaramillo, Juliana (2014). Muy tarde pero rentables: los ferrocarriles en Colombia durante el periodo 1920-1950. Cuadernos de Historia de Colombia, 34, 1-54.

Melgarejo-Rey, Florentino (1931). Informe del director de Educación al señor gobernador del departamento de Cundinamarca. Bogotá: Imprenta del Departamento.

República de Colombia (1957). Informe del Proyecto para el I Plan Quinquenal. Bogotá: República de Colombia.

Sáenz, Eduardo (2001). La Misión del Banco Mundial en Colombia, el Gobierno de Laureano Gómez (1950-1951) y la Asociación Nacional de Industriales (ANDI). Cuadernos de Economía, 20(35), 245-265.

Sánchez, Raúl (1967). Colombia, Planeamiento de la Educación. París: Unesco.

Valencia-Restrepo, Jorge (1987). La Misión Kemmerer y los intereses financieros británicos en Colombia durante la década de 1920. Lecturas de Economía, 24, 9-53. 